\title{
Novel identification of the ETS-1 splice variants p42 and p27 in prostate cancer cell lines
}

\author{
DAVID ADLER, JACQUELINE OCHSENFAHRT, KERSTIN FUCHS and NICOLAS WERNERT
}

Institute of Pathology, University Hospital Bonn, D-53127 Bonn, Germany

Received October 24, 2011; Accepted December 5, 2011

DOI: 10.3892/or.2012.1667

\begin{abstract}
ETS-1 is involved in cellular functions such as proliferation, migration, invasion, apoptosis and angiogenesis. The ETS-1 gene encodes three distinct proteins, ETS-1 p51 encoded by a full-length mRNA, ETS-1 p42 and ETS-1 p27 encoded by an alternatively spliced mRNA lacking exon VII and exons III-VI, respectively. ETS-1 p51, commonly considered to be the active form, has been studied in prostate cancer (PCa). However, the ETS-1 p42 and p27 variants have not yet been identified in PCa. Therefore, we aimed in this study at investigating whether the splice variants p42 and p27 are expressed in the androgen-dependent $\mathrm{VCaP}$ and $\mathrm{LNCaP}$ and the androgen-independent PC3 and DU-145 PCa cell lines. Using RT-PCR, we found the expression of both splice variants p42 and p27 at the mRNA level in the VCaP, LNCaP, PC3 and DU-145 PCa cell lines. We then confirmed the expression of ETS-1 p51 and its splice variants p42 and p27 at the protein level using an anti-ETS-1 antibody directed against the DNA-binding domain (DBD) in lysates prepared from the latter-mentioned cell lines, as well as in PC3 cell nuclear extract. Moreover, differences in the expression ratios of the ETS-1 splice variants within each cell line were also found. In conclusion, we have demonstrated for the first time the novel identification of the ETS-1 splice variants p42 and p27 in PCa cell lines. It is very likely that the role of ETS-1 p51 in PCa is significantly influenced by the presence of its splice variants ETS-1 p42 and p27 as competition, abundance, affinity, interactions and cross-talk among them will eventually determine the genes that will be targeted and subsequently affect cellular functions. Follow-up studies will need to address in functional terms, the roles of these splice variants in PCa cell lines, as well as their expression in PCa tissues and its correlation with clinical outcome.
\end{abstract}

Correspondence to: Professor Nicolas Wernert, Institute of Pathology, University Hospital Bonn, Sigmund-Freud-Str. 25, D-53127 Bonn, Germany

E-mail: nicolas.wernert@ukb.uni-bonn.de

Key words: ETS-1 p42, ETS-1 p27, transcriptional regulation, prostate cancer

\section{Introduction}

The ETS family of transcription factors which consists of 27 members in humans is characterized by an evolutionarily highly conserved DNA-binding domain, the ETS domain (1). ETS-1, the prototype of the ETS family is known to be involved in a broad variety of cellular functions (such as cell proliferation, migration, invasion, apoptosis and angiogenesis) (2). We have previously reported that ETS-1 promotes proliferation, migration or invasion of various neoplastic cell lines including PC3 prostate cancer cells, melanoma, HeLa, as well as glioma cells (3-6). Furthermore, ETS-1 has been reported to be overexpressed in latent and clinically manifest prostatic carcinomas and a strong expression of ETS-1 has been associated with poor tumor differentiation (7).

The ETS- 1 gene encodes three distinct proteins, ETS-1 p51 encoded by a full-length mRNA, ETS-1 p42 and ETS-1 p27 encoded by an alternatively spliced mRNA lacking exon VII and exons III-VI, respectively $(8,9)$.

The ETS-1 p42 splice variant (without exon VII) lacks an essential part of the autoinhibitory module and the calciumsensitive serines, which are phosphorylated upon calcium activation leading to the inhibition of DNA binding by reinforcing autoinhibtion $(2,10)$. Therefore, the lack of exon VII allows ETS-1 p42 to form stable complexes with DNA and contributes to the ability of the ETS-1 p42 protein to resist the negative action of calcium (2). ETS-1 p42 has been reported to bind more strongly to target DNA than ETS-1 p51 (11), commonly considered to be the active form, and to regulate different target genes in colon carcinoma cells (12). We have recently evaluated the functional effects of ETS-1 p42 in comparison to ETS-1 p51 expression in a well-characterized mouse fibroblast cell line, and found that ETS-1 p42 has different functions and target genes compared to ETS-1 p51 (e.g. TIMP-4, MMP-3, MMP-9 and MMP-13) (13).

On the other hand, the ETS-1 p27 splice variant (without exons III-VI) lacks the threonine-38 residue, the pointed (PNT) and the transactivation (TAD) domains affecting the transcriptional activity of ETS-1 and the transactivation of its target genes $(2,9)$. Interestingly, both inhibitory domains that surround the DNA-binding domain are conserved in ETS-1 p27, indicating that ETS-1 p27 is autoinhibited for DNA binding like the full length ETS-1 p51 (9).

The ETS-1 p51 protein and its splice variant p27 have been investigated in functional terms in mammary carcinoma 


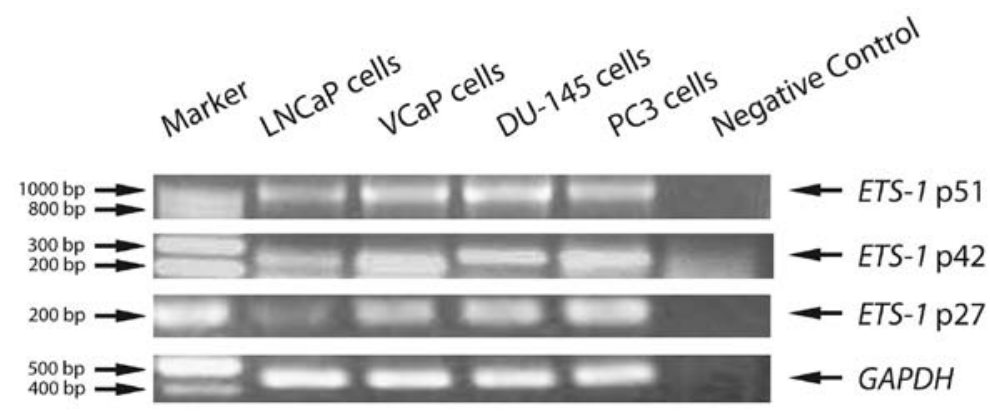

Figure 1. mRNA expression of ETS-1 and its splice variants in prostate cancer cell lines. RT-PCR analysis showing the full length ETS-1 p51 and its splice variants p42 and p27 in the androgen-dependent $\mathrm{LNCaP}$ and $\mathrm{VCaP}$, and the androgen-independent DU-145 and PC3 prostate cancer cell lines. The gene $G A P D H$ was used as a loading control, and amplification without a template was used as negative control.

cells (9). ETS-1 p27 has been shown to bind DNA in the same manner as ETS-1 p51 and to act both at a transcriptional and a subcellular level (9). More specifically, ETS-1 p27 has been shown to block ETS-1 p51-mediated transactivation of target genes in mammary carcinoma cells and to induce the translocation of ETS-1 p51 from the nucleus to the cytoplasm (9). Furthermore, the overexpression of ETS-1 p27s has been shown to repress the tumor properties of mammary carcinoma cells (9).

In PCa, the ETS family of transcription factors became a major focus of interest due to the frequent reporting of recurrent gene fusions occurring between the androgen-regulated prostate-specific serine protease TMPRSS2 gene, and several members of the ETS family such as ETV-1, ETV-4, ETV-5, and most commonly ERG (14-16). In previous studies, we were able to show that the ETS family prototype, ETS-1 is important for the biological properties of PCa cells (6), as well as the regulation of other ETS family members which might thus constitute a complex transcriptional regulatory network (17) that possibly involves the ETS-1 splice variants.

Since both ETS-1 p42 and p27 have not yet been investigated in PCa cell lines, we aimed in this study at examining whether the ETS-1 splice variants p42 and p27 are expressed in the androgen-dependent $\mathrm{VCaP}$ and $\mathrm{LNCaP}$ and the androgenindependent PC3 and DU-145 PCa cell lines.

\section{Materials and methods}

Cell culture. The prostate cancer cell lines LNCaP, VCaP, PC3 and DU-145 cells were grown in RPMI (Invitrogen, USA), DMEM (ATCC, USA), F12K (Invitrogen, USA) and MEM (Invitrogen, USA) media, respectively. The media were supplemented with $0.5 \%$ penicillin/streptomycin (Invitrogen) and $10 \%$ heat-inactivated fetal calf serum (Invitrogen). The MEM and RPMI media were additionally supplemented with $1 \%$ glucose (Invitrogen), $1 \%$ sodium pyruvate (Invitrogen) and $1 \%$ non-essential amino acids (NEIAA) (Invitrogen).

Preparation of cell lysates. Cell lysates were prepared from $\sim 5 \times 10^{6}$ cells as follows. Briefly, the pelleted cells were resuspended in a lysis buffer containing $50 \mathrm{mM}$ Tris-HCI, pH 8.0, $120 \mathrm{mM} \mathrm{NaCl}, 0.5 \% \mathrm{NP}-40$, and freshly added protease inhibitor (Complete Mini, Roche, Germany). The samples were then vortexed for $10 \mathrm{sec}$ and incubated on ice for $15 \mathrm{~min}$. Thereafter, the samples were centrifuged at $14,000 \times \mathrm{g}$ in a pre-cooled centrifuge for $10 \mathrm{~min}$ at $4^{\circ} \mathrm{C}$. The supernatants were then collected and labeled as cell lysates.

Western blot analysis. Following the determination of protein concentrations, the presence of ETS-1 p51, p42 and p27 in the cell lysates, and in PC3 cell nuclear extracts (Santa Cruz Biotechnology, Santa Cruz, USA) was determined by western blot analysis using rabbit polyclonal C-20 anti-ETS-1 antibody directed against the DNA-binding domain (DBD) (Santa Cruz Biotechnology). Furthermore, the housekeeping protein $\beta$-actin, an internal control was detected using rabbit polyclonal anti- $\beta$-actin antibody (Sigma-Aldrich, Germany).

RNA isolation and cDNA synthesis. RNA was isolated from the prostate cancer cell lines using the RNeasy Mini kit (Qiagen, Germany) as described by the manufacturer. The amount of the isolated RNA was then measured with the ND1000 Nanodrop (Peqlab, USA). Reverse transcription of total-RNA was performed with the SuperScript III First-Strand Synthesis SuperMix as described by the manufacturer (Invitrogen).

PCR. The polymerase chain reaction (PCR) was performed using the DNA-Engine Peltier Thermal Cycler (Bio-Rad Laboratories, Germany) to analyze the expression of ETS-1 p51, p42, p27 and the house-keeping gene GAPDH. The primers and sequences for ETS-1 p51, p27 and GAPDH (9), as well as ETS-1 p42 (13) were designed as previously described. All PCR products were analyzed on $2 \%$ agarose gels that were stained with ethidium bromide.

\section{Results}

mRNA expression of ETS-1 and its splice variants in prostate cancer cell lines. Using RT-PCR analysis, the mRNA expression of the full length ETS-1 p51 and its splice variants p42 and 27 was found in the androgen-dependent LNCaP and $\mathrm{VCaP}$, and the androgen-independent DU-145 and PC3 PCa cell lines (Fig. 1).

Protein expression of ETS-1 and its splice variants in prostate cancer cell lines. Western blot analysis using anti-ETS-1 antibody directed against the DNA-binding domain (DBD) (Santa Cruz Biotechnology) demonstrated that ETS-1 p51, p42 and p27 are expressed in PC3 cell nuclear extracts and in lysates prepared from the androgen-dependent $\mathrm{VCaP}$ and $\mathrm{LNCaP}$ and 

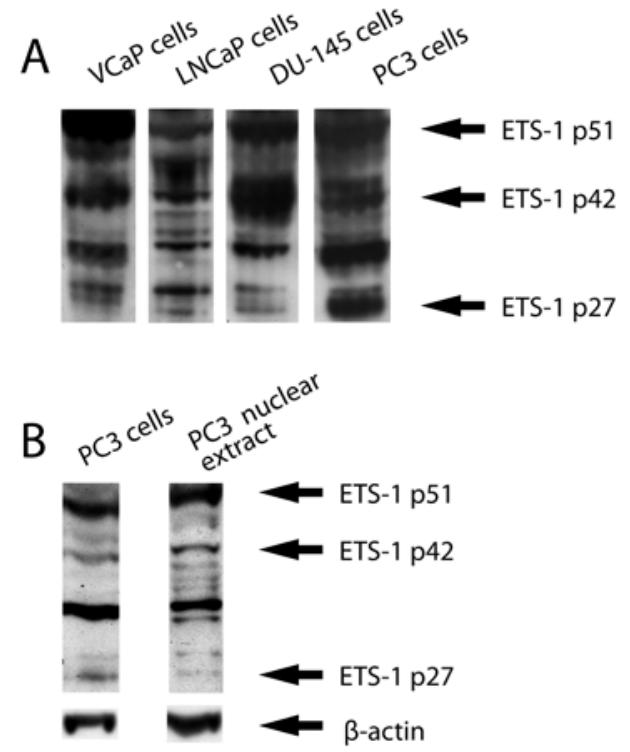

Figure 2. Protein expression of ETS-1 and its splice variants in prostate cancer cell lines. Western blot analysis using an anti-ETS-1 antibody directed against the DNA-binding domain (DBD) shows the protein expression of ETS-1 p51, p42 and p27 in VCaP, LNCaP, DU-145 and PC3 prostate cancer cell lines (A), and in PC3 cells/nuclear extract along with the housekeeping protein $\beta$-actin as a loading control (B).

the androgen-independent PC3 and DU-145 PCa cell lines (Fig. 2).

A strong band of $\sim 35 \mathrm{kDa}$ was also detected, and it is likely to correspond to another splice variant of ETS-1 (without exons IV and VII) (18), which has not yet been reported to result in the generation of a protein (Fig. 2). Additionally, the several weak bands that were also detected are very likely to be the phosphorylated forms of ETS-1 (Fig. 2).

\section{Discussion}

ETS-1 overexpression has been associated with latent and clinically manifest prostatic carcinomas, as well as poor tumor differentiation (7). In previous studies, we found that ETS-1 is involved in a variety of cellular processes (e.g. proliferation, migration and invasion) of several neoplastic cell lines such as, melanoma, HeLa, glioma cells as well as PC3 prostate cancer cells (3-6). In PC3 cells, ETS-1 promotes their migration and regulates the expression of many genes involved in important steps of the so-called metastatic cascade $(6,19)$.

Besides ETS-1 p51, commonly considered to be the active form, which is encoded by a full-length mRNA, ETS-1 encodes two other distinct proteins, ETS-1 p42 and p27, which are encoded by an alternatively spliced mRNA lacking exon VII and exons III-VI, respectively $(8,9)$. These two splice variants have been studied by us and others in functional terms in cell lines such mouse fibroblasts and mammary carcinoma cells as described above (9,11-13). However, both splice variants have not yet been investigated in prostate cancer cell lines.

Therefore, we investigated in this study whether the ETS-1 splice variants p42 and p27 are expressed in the androgendependent $\mathrm{VCaP}$ and $\mathrm{LNCaP}$ and the androgen-independent PC3 and DU-145 PCa cell lines.
Using RT-PCR, we observed the expression of both splice variants p42 and p27 at the mRNA level in the androgendependent $\mathrm{VCaP}$ and $\mathrm{LNCaP}$ and the androgen-independent PC3 and DU-145 PCa cell lines (Fig. 1).

We then confirmed the expression of ETS-1 p51 and its splice variants p42 and p27 at the protein level using an antiETS-1 antibody directed against the DNA-binding domain (DBD) in lysates prepared from the androgen-dependent $\mathrm{VCaP}$ and LNCaP and the androgen-independent PC3 and DU-145 PCa cell lines (Fig. 2). All 3 proteins were also expressed in PC3 cell nuclear extracts (Fig. 2).

Notably, a strong band of approximately $35 \mathrm{kDa}$ was also detected in the lysates as well as in the nuclear extract, and it is likely to correspond to another splice variant of ETS-1 (without exons IV and VII) (18), which has not yet been reported to result in the generation of a protein (Fig. 2). The several weak bands that were detected close to the molecular weight area of ETS-1 p42 and p27 are very likely to be the phosphorylated forms of ETS-1, as ETS-1 is commonly regulated by phosphorylation (2) (Fig. 2).

Our results also show that there are differences in the expression ratios of the full length ETS-1 protein and of the ETS-1 splice variants within each cell line. These differences are most likely due to the different biological properties of these cell lines. The DU-145, PC3, LNCaP and VCaP cell lines are derived from different origins, brain metastasis, an advanced androgen-independent bone metastasis, a supraclavicular lymph node metastasis and a metastatic lesion to a lumbar vertebral body of a patient with hormone refractory prostate cancer, respectively.

It is worth noting that the differences in the expression ratios of the ETS-1 splice variants within each cell line is highly interesting in light of a previous study demonstrating that an increase in the ETS-1 p27/ETS-1 p51 ratio induces the delocalization of ETS-1 from the nucleus to the cytoplasm in mammary carcinoma cells (9). Therefore, the ratios that we found of the ETS-1 splice variants might also be significant in $\mathrm{PCa}$, in the sense that they reflect their mechanistic complexity, competition among splice variants based on their abundance, affinity, co-factors and genes that may be regulated by different ETS-1 proteins in PCa.

Our preliminary findings about the expression level of a specific ETS-1 splice variant in a PCa cell line may provide us in further studies with insights into the molecular mechanisms of action of these splice variants in $\mathrm{PCa}$, due to the already known unique properties of each splice variant $(8,9)$, and their different target genes $(9,12,13)$. For instance, in comparison to ETS-1 p51, ETS-1 p42 lacks an essential part of the autoinhibitory module and the calcium-sensitive serines, which are phosphorylated upon calcium activation leading to the inhibition of DNA binding by reinforcing autoinhibition $(2,10)$, whereas the ETS-1 p27 lacks the threonine-38 residue, the pointed (PNT) and the transactivation (TAD) domains affecting the transcriptional activity of ETS-1 and the transactivation of its target genes $(2,9)$.

Taken together, we have demonstrated for the first time the novel identification of the ETS-1 splice variants p42 and p27 in PCa cell lines. It is very likely that the role of ETS-1 $\mathrm{p} 5$, commonly considered being the active form, in $\mathrm{PCa}$ is significantly influenced by the presence of its splice variants 
ETS-1 p42 and p27, as competition, abundance, affinity, interactions, and cross-talk among them will eventually determine the genes that will be targeted and subsequently affect cellular functions. Therefore, follow-up studies will address in functional terms, the roles of these ETS-1 splice variants in $\mathrm{PCa}$ cell lines, as well as their expression in prostate cancer tissues and their correlation with clinical outcome.

\section{Acknowledgements}

We are grateful to the DFG(Deutsche Forschungsgemeinschaft; German Research Association, grant no. WE 1104/11-1) and the German Cancer Aid (Deutsche Krebshilfe, grant no. 107827) for their financial support.

\section{References}

1. Hollenhorst PC, Jones DA and Graves BJ: Expression profiles frame the promoter specificity dilemma of the ETS family of transcription factors. Nucleic Acids Res 32: 5693-5702, 2004.

2. Dittmer J: The biology of the Ets1 proto-oncogene. Mol Cancer 2: 29,2003

3. Rothhammer T, Hahne JC, Florin A, et al: The Ets-1 transcription factor is involved in the development and invasion of malignant melanoma. Cell Mol Life Sci 61: 118-128, 2004.

4. Hahne JC, Okuducu AF, Kaminski A, Florin A, Soncin F and Wernert N: Ets-1 expression promotes epithelial cell transformation by inducing migration, invasion and anchorage-independent growth. Oncogene 24: 5384-5388, 2005.

5. Sahin A, Vercamer C, Kaminski A, et al: Dominant-negative inhibition of Ets 1 suppresses tumor growth, invasion and migration in rat C6 glioma cells and reveals differentially expressed Ets 1 target genes. Int J Oncol 34: 377-389, 2009.

6. Adler D, Langer B, Lindstrot A, et al: Ets-1 is implicated in the regulation of androgen co-regulator FHL2 and reveals specificity for migration, but not invasion, of $\mathrm{PC} 3$ prostate cancer cells. cells. Oncol Rep 25: 1125-1129, 2011.
7. Alipov G, Nakayama T, Ito M, et al: Overexpression of Ets-1 proto-oncogene in latent and clinical prostatic carcinomas. Histopathology 46: 202-208, 2005.

8. Koizumi S, Fisher RJ, Fujiwara S, et al: Isoforms of the human ets-1 protein: generation by alternative splicing and differential phosphorylation. Oncogene 5: 675-681, 1990.

9. Laitem C, Leprivier G, Choul-Li S, et al: Ets-1 p27: a novel Ets-1 isoform with dominant-negative effects on the transcriptional properties and the subcellular localization of Ets-1 p51. Oncogene 28: 2087-2099, 2009.

10. Rabault B and Ghysdael J: Calcium-induced phosphorylation of ETS1 inhibits its specific DNA binding activity. J Biol Chem 269: 28143-28151, 1994.

11. Fisher RJ, Fivash M, Casas-Finet J, et al: Real-time DNA binding measurements of the ETS1 recombinant oncoproteins reveal significant kinetic differences between the p42 and p51 isoforms. Protein Sci 3: 257-266, 1994.

12. Li R, Pei H and Papas T: The p42 variant of ETS1 protein rescues defective Fas-induced apoptosis in colon carcinoma cells. Proc Natl Acad Sci USA 96: 3876-3881, 1999.

13. Hahne JC, Fuchs T, Florin A, Edwards D, Pourtier A, Soncin F and Wernert N: Evaluation of effects caused by differentially spliced Ets-1 transcripts in fibroblasts. Int J Oncol 39: 1073-1082, 2011.

14. Tomlins SA, Rhodes DR, Perner S, et al: Recurrent fusion of TMPRSS2 and ETS transcription factor genes in prostate cancer. Science 310: 644-648, 2005.

15. Rubin MA, Maher CA and Chinnaiyan AM: Common gene rearrangements in prostate cancer. J Clin Oncol 29: 3659-3668, 2011.

16. Kumar-Sinha C, Tomlins SA and Chinnaiyan AM: Recurrent gene fusions in prostate cancer. Nat Rev Cancer 8: 497-511, 2008.

17. \$ GOH' , Lindstrot A, Langer B, Buettner R and : HOHWM Differential expression of ETS family members in prostate cancer tissues and androgen-sensitive and insensitive prostate cancer cell lines. Int J Mol Med 28: 89-93, 2011.

18. Jorcyk CL, Watson DK, Mavrothalassitis GJ and Papas TS: The human ETS1 gene: genomic structure, promoter characterization and alternative splicing. Oncogene 6: 523-532, 1991.

19. Adler D, Lindstrot A, Langer B, Buettner R and Wernert N: Comprehensive gene expression microarray analysis of Ets-1 blockade in PC3 prostate cancer cells and correlations with prostate cancer tissues: Insights into genes involved in the metastatic cascade. Int J Mol Med 27: 811-819, 2011. 